\title{
APPLYING THE CONSERVATION OF MASS PRINCIPLE FOR THE INTERNAL VALIDATION OF FLOW MEASUREMENTS IN CARDIOVASCULAR MAGNETIC RESONANCE IMAGING
}

\author{
Judy Rizk, Eman Elsharkawy, Gihan Magdy, Tarek Elzawawy
}

Department of Cardiology, Faculty of Medicine, Alexandria University, Alexandria, Egypt

\begin{abstract}
Background: Flow quantification is a central component in the evaluation of structural heart disease. Given the acoustic limitations of echocardiography, two-dimensional phase contrast magnetic resonance imaging (MRI) may be needed for non-invasive flow quantification. Careful evaluation of the dataset is necessary to ensure that the flow measurements are reliable.

Aim: The aim of this study was to use the conservation of mass principle to check the validity of the flow measurements.

Methods: Twenty consecutive patients referred cardiac MRI were included. Scans were acquired using a standard cardiac 1.5 Tesla MRI scanner. Phase-contrast MRI flow acquisition was planned in the proximal main pulmonary artery (MPA) and in the ascending aorta (Asc Ao).

Results: Net flow in the MPA was $75 \pm 17 \mathrm{ml}$ and net aortic flow was 74 $\pm 18 \mathrm{ml}(\mathrm{P}=0.565)$. Bland-Altman analysis showed a mean difference between measurements of $1.45 \pm 11.0 \mathrm{ml}$.

Conclusion: Applying the conservation of mass principle to check the internal validity of flow data is feasible and serves as a quality control measure for cardiac MRI.
\end{abstract}

Keywords: Glomerular conservation of mass; magnetic resonance imaging; flow quantification

\section{INTRODUCTION}

Flow quantification is a central component in the evaluation of structural heart disease. Doppler echocardiography allows non invasive flow assessment and is the initial imaging modality of choice. ${ }^{(1)}$ However, accurate alignment of the Doppler beam with flow is necessary and flow volume calculation is based on the assumption of circular crosssectional orifice area. ${ }^{(2)}$ Moreover, it is susceptible to acoustic window limitations. ${ }^{(3)}$ Two-dimensional (2D) phase contrast (PC) magnetic

resonance imaging (MRI) and can be used to measure blood flow velocity and volume through a pre-defined plane. ${ }^{(4)}$ It has the advantage of unlimited imaging window and fewer flow assumptions as well as being a component of a MRI study that is considered the gold standard for ventricular volume quantification. ${ }^{(5)}$ Cardiac catheterization is an invasive procedure associated with radiation exposure and the use of iodinated contrast. Therefore, it is mainly reserved when the calculation of pulmonary vascular resistance or the measurement of chamber intra-cavity pressure is necessary as well as for percutaneous therapeutic interventions. $^{(1,3,6)}$

Several technical factors should be considered during acquisition to obtain accurate flow measurements. During planning the acquisition, alignment of the imaging plane perpendicular to the vessel long axis is necessary. The vessel of interest should be placed at the scanner isocenter. The velocity encoding (venc) should be selected according to the expected peak velocity; a too high setting will increase noise and a too low setting will result in aliasing and flow misinterpretation. Finally, correct ECG-triggering during acquisition should be monitored. ${ }^{(7-9)}$

PC-MRI is susceptible to sources of error that can result in inaccurate flow measurements. Phase offset errors occur due to eddy currents and concomitant gradient fields. ${ }^{(9)}$ Correction of background phase errors can be done using routine phantom calibration or using data preprocessing software to subtract phase offset from static tissue. ${ }^{(4,10)}$ Partial volume averaging is also another source of systematic error. ${ }^{(4)}$ This can be minimized by increasing the spatial resolution in the acquisition parameters and by proper alignment of the imaging plane. ${ }^{(9)}$ Through-plane 
motion of the heart can further impact measured flow volumes. ${ }^{(9)}$ In addition, in the presence complex flow patterns, flow volumes vary with the anatomical level of the measurement plane. ${ }^{(11)}$

Precise vessel wall definition during post-processing is important obtain accurate flow measurements. Due to vessel wall motion during the cardiac cycle, vessel wall contour should be reviewed in each image. ${ }^{(4,7)}$

Although the implementation of acquisition and interpretation guidelines described above helps avoid many sources of error, ${ }^{(4,7,8)}$ the dataset should always be carefully examined to ensure that the flow measurements are reliable.

The aim of this study was to use the conservation of mass principle to check the validity of flow measurements.

\section{METHODS}

Twenty consecutive patients referred for cardiac MRI were included. The study was approved by the Institutional Ethics Committee.

Scans were acquired using a standard cardiac 1.5 Tesla MRI scanner. Phase-contrast MRI acquisition was planned in the proximal main pulmonary artery (MPA) and the proximal ascending aorta (Asc Ao) perpendicular to the vessel long axis in two orthogonal planes. Velocity encoding was set at $25 \%$ above the expected peak velocity. Steady state free precession cines were acquired for ventriculography.

Standard postprocessing software was used to measure ventricular volumes and function and quantify flow.

\section{STATISTICAL ANALYSIS}

Continuous variables were expressed as mean \pm standard deviation. Paired t-test was used to compare net aortic and net pulmonary flow as well as with ventricular stroke volumes. Agreement was assessed using Bland-Altman analysis and expressed as mean difference and limits of agreement $( \pm 1.96$ standard deviation). Correlation was assessed using Pearson's correlation. Statistical significance was defined as two-sided $P<0.05$. Statistical analysis was performed using Graphpad Prism 5 (Graphpad Software, Inc., LA Jolla, CA, USA).

\section{RESULTS}

All subjects had regular cardiac rhythm during the scan. The duration of the acquisition of the 2D PC-MRI 9.5

Net flow in the MPA was $75 \pm 17 \mathrm{ml} /$ cycle and net aortic flow was $74 \pm 18 \mathrm{ml} /$ cycle $(\mathrm{P}=0.565)$.

Bland-Altman analysis showed a mean difference between measurements of $1.45 \pm 11.0 \mathrm{ml}$ (Figure 1a). Significant correlation between net flow in both vessels was present (Pearson $\mathrm{r}=0.799, \mathrm{P}<0.0001$ ) as shown in Figure $1 b$.

Similarly good agreement was present between left ventricular stroke volume and aortic flow $(69.45 \pm 15.45$ and $73.70 \pm 17.71 \mathrm{ml}$ respectively, $\mathrm{P}=0.073)$. BlandAltman analysis showed a mean difference of $4.25 \pm 9.99$ $\mathrm{ml}$ with significant correlation (Pearson $\mathrm{r}=0.827, \mathrm{P}<$ 0.0001) (Figure 2).

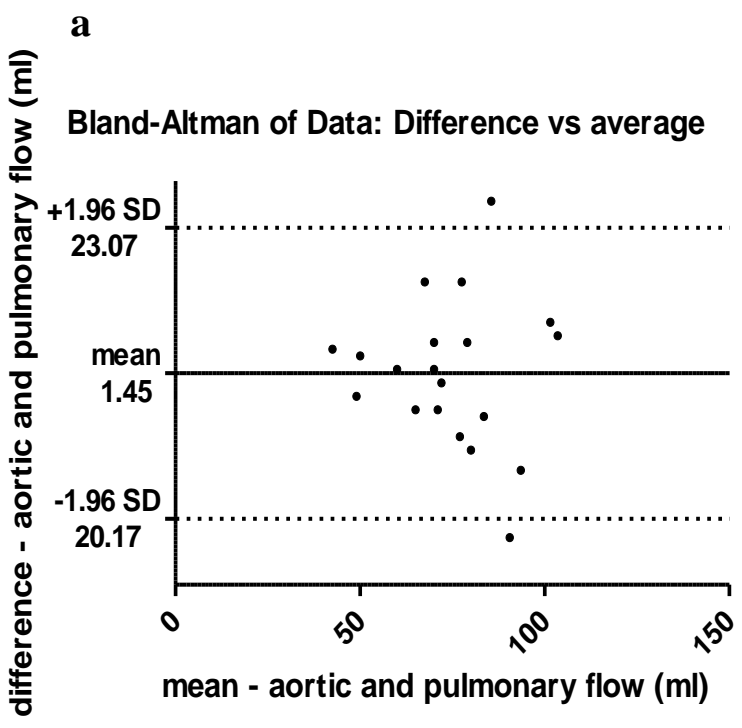

b

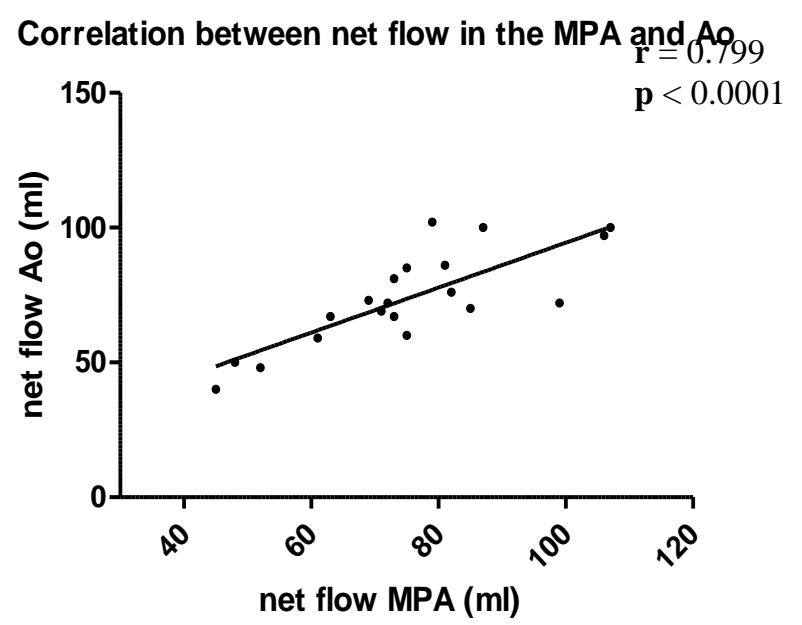

Figure (1): Bland-Altman plot of the difference between net flow in the aorta and the MPA presented as difference against the mean. Limits of agreement $( \pm 1.96 \mathrm{SD})$ are shown as dashed lines (a). Correlation between net flow in the aorta and MPA (b). $\mathrm{Ao}=$ aorta, $\mathrm{MPA}=$ main pulmonary artery 
a

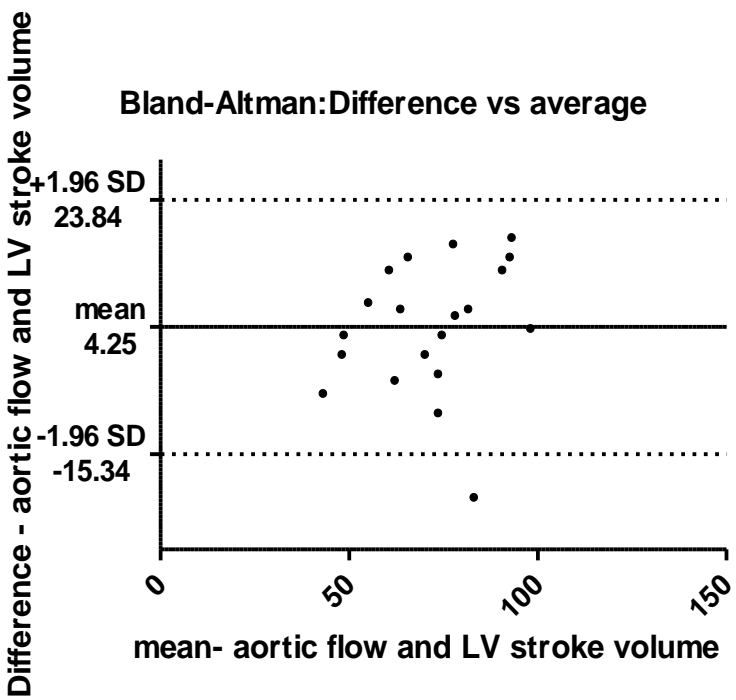

b

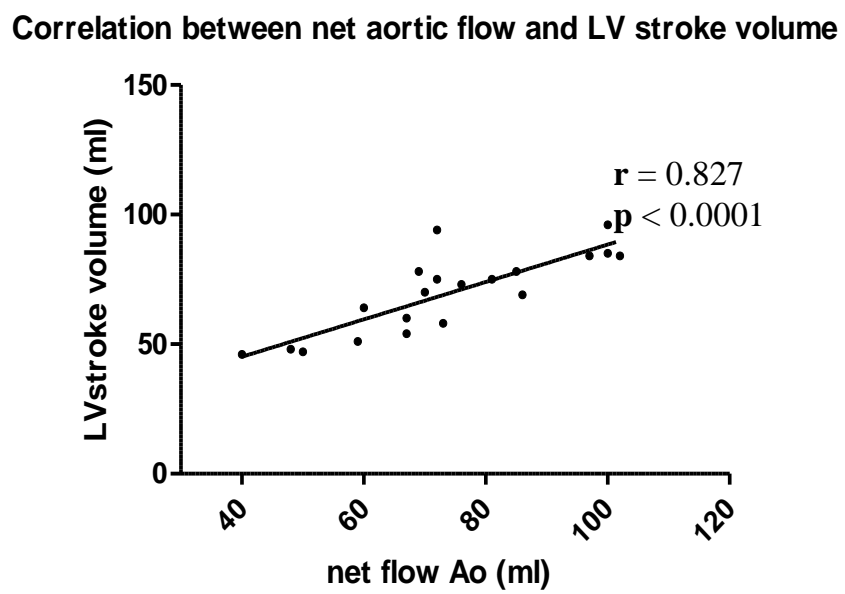

Figure (2): Bland-Altman plot of the difference between net aortic flow and LV stroke volume presented as difference against the mean. Limits of agreement $( \pm 1.96 \mathrm{SD})$ are shown as dashed lines (a). Correlation between net flow aortic flow and LV stroke volume (b). Ao = aorta, $\mathrm{LV}=$ left ventriclel

\section{DISCUSSION}

The The main finding in this study is using the agreement between net aortic and pulmonary flow acquired with PCMRI and ventricular stroke volume by planimetry to check the validity of flow measurements.

Based on the conservation of mass principle, the internal consistency of net aortic and pulmonary flow can be used to ensure the validity of flow measurements. In the absence of shunt, net aortic flow and net pulmonary flow suffice for validation. Agreement of net flow through the MPA and the sum of flow through the right and left pulmonary arteries can be used in patients with congenital heart disease.

As flow measurements are susceptible to several limitations described above, assessing the internal consistency of the data enables the detection of any error. When incongruous data are present, phase images should be inspected for aliasing and the vessel wall trace should be revised. It is also important to review the heart rhythm during the study for any irregularity resulting in different cardiac cycle lengths and stroke volumes. Clinical patient information as in congenital heart disease may indicate the presence of shunt or collateral flow.

Forward pulmonary and aortic flow should also correspond to the corresponding ventricular stroke volume by planimetry. This can also serve as an additional quality control measure. It is essential to consider the presence of valvular regurgitation in the interpretation. In fact, atrioventricular valvular regurgitation can be indirectly quantified using ventricular stroke volume and forward semilunar valvular flow. ${ }^{(5)}$
4D flow MRI has emerged as a development in the field of MRI that enables the comprehensive study of flow. ${ }^{(12)}$ It involves the acquisition of a three-dimensional timeresolved PC-MRI volume with velocity-encoding in all three spatial directions along the cardiac cycle. ${ }^{\left({ }^{3}\right)}$ This has the advantage of allowing flexible retrospective analysis plane placement at any desired location, as opposed to 2D-PC MRI that requires the individual planning of each analysis plane during scan acquisition. In addition, the valve tracking feature of 4D flow MRI overcomes through-plane annular motion resulting in more accurate flow quantification. ${ }^{(14)}$ However, the main limitation of 4D flow MRI is the trade-off between scan time and spatiotemporal resolution. ${ }^{(13,15)}$ Therefore, in spite of the promising advantages of 4D flow CMR, its use for flow measurement in routine clinical practice is not yet standardized and it is still mainly a research tool.

In conclusion, this study shows that applying the conservation of mass principle to check the internal validity of PC-MRI flow data is feasible. It should be used as a quality control measure for cardiac MRI studies for both the flow measurements as well as ventriculography.

\section{REFERENCES}

1. Di Salvo G, Miller O, Babu Narayan S, Li W, Budts W, Valsangiacomo Buechel ER, et al. Imaging the adult with congenital heart disease: a multimodality imaging approach-position paper from the EACVI. Eur Heart J Cardiovasc Imaging. 2018;19(10):1077-98.

2. Quiñones MA, Otto CM, Stoddard M, Waggoner A, Zoghbi WA. Recommendations for quantification of Doppler echocardiography: A report from the Doppler quantification task force of the nomenclature and 
tandards committee of the American Society of Echocardiography. J Am Soc Echocardiogr. 2002;15(2):167-84.

3. Babu-Narayan SV, Giannakoulas G, Valente AM, Li W, Gatzoulis MA. Imaging of congenital heart disease in adults. Eur Heart J. 2016;37(15):1182-95.

4. Fratz S, Chung T, Greil GF, Samyn MM, Taylor AM, Valsangiacomoechel ER, et al. Guidelines and protocols for cardiovascular magnetic resonance in children and adults with congenital heart disease: SCMR expert consensus group on congenital heart disease. J Cardiovasc Magn Reson. 2013;15(1):51.

5. Zoghbi WA, Adams D, Bonow RO, Enriquez-Sarano M, Foster E, Grayburn PA, et al. Recommendations for Noninvasive Evaluation of Native Valvular Regurgitation: A Report from the American Society of Echocardiography Developed in Collaboration with the Society for Cardiovascular Magnetic Resonance. J Am Soc Echocardiogr. 2017;30(4):303-71.

6. Burchill LJ, Huang J, Tretter JT, Khan AM, Crean AM, Veldtman GR, et al. Noninvasive Imaging in Adult Congenital Heart Disease. Circ Res. 2017;120(6):995-1014.

7. Schulz-Menger J, Bluemke DA, Bremerich J, Flamm SD, Fogel MA, Friedrich MG, et al. Standardized image interpretation and post processing in cardiovascular magnetic resonance: Society for Cardiovascular Magnetic Resonance (SCMR) Board of Trustees Task Force on Standardized Post Processing. J Cardiovasc Magn Reson. 2013;15(1):35.

8. Kramer CM, Barkhausen J, Flamm SD, Kim RJ, Nagel E. Standardized cardiovascular magnetic resonance (CMR) protocols 2013 update. J Cardiovasc Magn Reson. 2013;15(1):91.

9. Nayak KS, Nielsen JF, Bernstein MA, Markl M, P DG, R $\mathrm{MB}$, et al. Cardiovascular magnetic resonance phase contrast imaging. J Cardiovasc Magn Reson. 2015;17:71.

10. Holland BJ, Printz BF, Lai WW. Baseline correction of phase-contrast images in congenital cardiovascular magnetic resonance. J Cardiovasc Magn Reson. 2010;12 (1): 11 .

11. Nordmeyer S, Riesenkampff E, Messroghli D, Kropf S, Nordmeyer J, Berger F, et al. Four-dimensional velocityencoded magnetic resonance imaging improves blood flow quantification in patients with complex accelerated flow. J Magn Reson Imaging. 2012;37(1):208-16.

12. Markl M, Kilner PJ, Ebbers T. Comprehensive 4D velocity mapping of the heart and great vessels by cardiovascular magnetic resonance. J Cardiovasc Magn Reson. 2011;13(1):7.

13. Dyverfeldt $\mathrm{P}$, Bissell M, Barker AJ, Bolger AF, Carlhäll C-J, Ebbers T, et al. 4D flow cardiovascular magnetic resonance consensus statement. J Cardiovasc Magn Reson. 2015;17(1):72.

14. Westenberg JJM, Roes SD, Ajmone Marsan N, Binnendijk NMJ, Doornbos J, Bax JJ, et al. Mitral Valve and Tricuspid Valve Blood Flow: Accurate Quantification with 3D Velocity-encoded MR Imaging with Retrospective Valve Tracking. Radiology. 2008; 249(3):792-800.

15. Markl M, Schnell S, Barker AJ. 4D flow imaging: current status to future clinical applications. Curr Cardiol Rep. 2014;16(5):481 